\title{
Porous Silicon Based Rugate Filter Wheel for Multispectral Imaging Applications
}

\author{
Shervin Keshavarzi, ${ }^{1, *, z}$ Andras Kovacs, ${ }^{1}$ Mohammad Abdo, ${ }^{2}$ Vlad Badilita, ${ }^{2}$ Rui Zhu, ${ }^{1}$ \\ Jan G. Korvink, ${ }^{2}$ and Ulrich Mescheder ${ }^{1}$
}

${ }^{l}$ Department of Medical and Mechanical Engineering, Furtwangen University, Institute for Microsystems

Technology-IMST, Furtwangen 78120, Germany

${ }^{2}$ Karlsruhe Institute of Technology-KIT, Institute of Microstructure Technology-IMT, Karlsruhe 76131, Germany

\begin{abstract}
Porous silicon based rugate filters are emerging as interesting functionalized optical components in Micro-Opto-Electro-Mechanical Systems due to their specific nanostructures and superior optical properties. In this work, a cost-effective approach to generate a porous silicon based rugate filter wheel containing nine filter segments in the visible wavelength range suitable for multispectral imaging systems is presented. The filter wheel segments are patterned on a silicon wafer using silicon nitride insulating masks and generated using the anodization technique. Specific characteristics of filter segments are adjusted by the current squeezing effect during the anodization through the geometrical size of the filter segments, which results in local current redistributions and consequently porous silicon formation rate and porosity modifications. A finite element model made in COMSOL Multiphysics is also presented to study redistributions of the current density and the current squeezing effect during the anodization for the proposed filter wheel. The proposed filter wheel can be miniaturized and integrated into a portable multispectral imaging system.

(C) The Author(s) 2019. Published by ECS. This is an open access article distributed under the terms of the Creative Commons Attribution 4.0 License (CC BY, http://creativecommons.org/licenses/by/4.0/), which permits unrestricted reuse of the work in any medium, provided the original work is properly cited. [DOI: 10.1149/2.0251902jss]

(c)) BY
\end{abstract}

Manuscript submitted October 16, 2018; revised manuscript received February 28, 2019. Published March 20, 2019.

Due to its optical and electrical properties, porous silicon (PSi) has become an interesting and superior candidate for chemical and biological sensing applications. ${ }^{1-3}$ The adsorption of chemical or biological substances into the pores changes the electrical ${ }^{4}$ and optical ${ }^{5}$ properties of PSi, allowing convenient and sensitive measurement of concentrations of liquids or gases. ${ }^{2}$ The high internal surface area $\left(>500 \mathrm{~m}^{2} / \mathrm{cm}^{3}\right)^{6}$ of PSi can provide a suitable immobilization of an increased amount of various biomolecules, such as enzymes, ${ }^{7}$ DNA fragments, ${ }^{8}$ and antibodies ${ }^{9}$ in a relatively small area.

Several types of optical biosensors operating with PSi-based optical elements, such as Fabry-Perot layers, ${ }^{10}$ Bragg reflectors, ${ }^{11}$ rugate filters, ${ }^{12}$ microcavities, ${ }^{13}$ waveguides, ${ }^{14}$ photonic crystals,${ }^{15}$ and diffraction gratings ${ }^{16}$ have been so far reported. Among them, rugate filters are a class of optical filters, which are characterized by a sinusoidal continuous variation of the refractive index in a direction perpendicular to the plane of the filter. ${ }^{17}$ The reflectance spectrum of a rugate filter shows a high reflectivity around a filter specific wavelength and a very low reflectivity elsewhere.

One of the recent applications of PSi rugate filters is the use of such filters in spectral imaging systems. ${ }^{18}$ Spectral imaging is an emerging non-invasive, non-destructive, and contact-free analysis technique that provides spatially-resolved spectral information on a target in the form of a 3D data set. ${ }^{19}$ Public health is one of the fields where spectral imaging can play a vital role; as a tool for early detection of diseases and rapid medical diagnosis ${ }^{20}$ and for monitoring of the safety and quality of food products. ${ }^{21}$ To reveal this potential, a portable lowcost spectral imaging system is needed to allow physicians to detect diseases in their clinics, or patients to self-monitor their conditions, and consumers to maintain healthy food intakes. However, the large size, high instrumentation effort, and high costs continue to prevent the wide scale adoption of spectral imaging.

In this work, we present a cost-effective approach to generate a PSi rugate filter wheel containing nine filter segments in the visible wavelength range (570-820 nm) suitable for wavelength scanning multispectral imaging systems. ${ }^{22}$ In this approach, the filter wheel segments are patterned on a $\mathrm{Si}$ wafer covered by thin silicon nitride isolation layers and generated using the anodization technique. Specific characteristics of filter segments are adjusted by the current squeezing effect during the anodization through the geometrical size of the filter segments. ${ }^{23-25}$ A finite element model developed in COMSOL Multiphysics is also presented to study the influence of a filter segment size

\footnotetext{
*Electrochemical Society Student Member
}

${ }^{\mathrm{z}}$ E-mail: kesh@hs-furtwangen.de (opening area) and its surrounding masking area on redistribution of the current density, and the current squeezing during the anodization for the proposed filter wheel. The approach can also be adapted to create miniaturized rugate filter wheels convenient for portable multispectral imaging systems since PSi properties, especially the formation rates, are defined by the applied current densities (current divided by the anodization area) for a specific wafer resistivity range and type, and electrolyte used. Hence, down scaling of the filter wheel can be performed by adjusting the current. By downscaling and thinning, the filter chip can be integrated into a portable multispectral imaging system.

\section{Theory}

A rugate filter is essentially a Distributed Bragg Reflector (DBR) in which the refractive index of the material is sinusoidally varied in a direction perpendicular to plane of the filter. ${ }^{26}$ The refractive index profile $(n(x))$ of a rugate filter centered at resonance peak wavelength $\left(\lambda_{0}\right)$ is classically written as: ${ }^{17,27,28}$

$$
n(x)=n_{0}+\frac{\Delta n}{2} \sin \left(\frac{4 \pi x}{\lambda_{0}}\right)
$$

where $x$ is the distance from the filter surface (optical path length), $n_{0}$ is the average refractive index, and $\Delta n=n_{H}-n_{L}$ is the refractive index contrast between two successive layers with high refractive index $\left(n_{H}\right)$ and low refractive index $\left(n_{L}\right)$.

In general, the resonance peak wavelength $\left(\lambda_{0}\right)$ of a rugate filter is analogous to a quarter-wave stack DBR and is usually approximated by: 28,29

$$
\frac{\lambda_{0}}{4}=n_{H} d_{H}=n_{L} d_{L}
$$

where $d_{H}$ and $d_{L}$ are thicknesses of high and low refractive index layers, respectively.

The difference between a rugate filter and a DBR is the bandgap of the filter, which is slightly narrower (by a factor of $\pi / 4$ ) than the bandwidth of a quarter-wave stack DBR. ${ }^{28}$ Additionally, higher harmonics can be completely suppressed in rugate filters if the condition $\left(n_{H} d_{H}=n_{L} d_{L}\right)$ is held. The reflectance $(R)$ of a rugate filter at the resonance peak wavelength is dependent on number of filter stack (layer) 


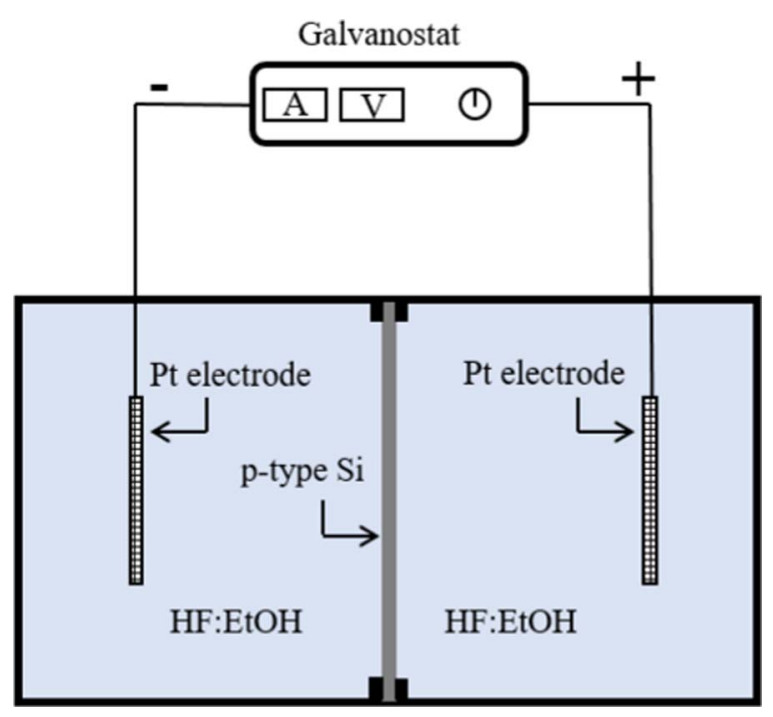

Figure 1. 2D schematic of anodic etching of silicon in a HF based electrolyte in a double cell anodization tank.

and usually estimated by: ${ }^{30}$

$$
R \approx\left|\frac{1-\left(\frac{n_{H}}{n_{L}}\right)^{\frac{m \pi}{2}}}{1+\left(\frac{n_{H}}{n_{L}}\right)^{\frac{m \pi}{2}}}\right|^{2} \times 100 \%
$$

where $m$ is the number of layers (periods).

The refractive index spectrum of a rugate filter (Eq. 2) shows smallamplitude higher-order harmonic peaks. However, to completely suppress these higher-order harmonics, the logarithm of the refractive index profile needs to be modulated sinusoidally as follows: $:^{28,31}$

$$
\log [n(x)]=\frac{\log \left(n_{H}\right)+\log \left(n_{L}\right)}{2}+\frac{\log \left(n_{H}\right)-\log \left(n_{L}\right)}{2} \sin \left(\frac{4 \pi x}{\lambda_{0}}\right)
$$

When the difference between $n_{H}$ and $n_{L}$ is very small, Eq. 4 tends to Eq. 1, and such a sinusoidal profile results in a reflection spectrum showing a single stop-band at $\lambda_{0}{ }^{28}$ However, because of the sharp truncation of the refractive index modulation at the boundaries of the filter, this simple sinusoidal index profile additionally produces side lobes on both sides of the stop-band. ${ }^{17}$ These undesired side lobes can be suppressed by employing additional processes such as index matching and apodization. ${ }^{17,28,32}$

A rugate filter based on porous silicon can be typically fabricated by applying a sinusoidal current density profile during the anodization process. ${ }^{33-35}$ This results in a continuous variation of the porosity of the generated PSi, and consequently a continuous variation of the refractive index. A PSi is typically made by electrochemical etching (anodization) of a crystalline silicon ( $\mathrm{Si}$ ) in a hydrofluoric acid (HF) based solution in a double cell anodization tank (see Fig. 1). ${ }^{36}$ The anodization current density, doping level of $\mathrm{Si}$, and concentration of HF determine the porosity and pore size of the PSi. ${ }^{37}$ The higher the porosity, the lower is the refractive index of the PSi (see Fig. 2). ${ }^{38}$ The corresponding effective refractive index $\left(n_{P S i}\right)$ of a PSi layer can be typically estimated through the Bruggeman effective medium approximation relationship given in the case of a two media approach by: ${ }^{39-41}$

$$
P_{P S i} \frac{n_{a i r}{ }^{2}-n_{P S i}^{2}}{n_{a i r}{ }^{2}+2 n_{P S i}{ }^{2}}+\left(1-P_{P S i}\right) \frac{n_{S i}^{2}-n_{P S i}^{2}}{n_{S i}{ }^{2}+2 n_{P S i}{ }^{2}}=0
$$

Here, $n_{\text {air }}, n_{S i}$, and $n_{P S i}$ are respectively the refractive indexes of air (pores), silicon and porous silicon, and $P_{P S i}$ is the porosity of the PSi layer.

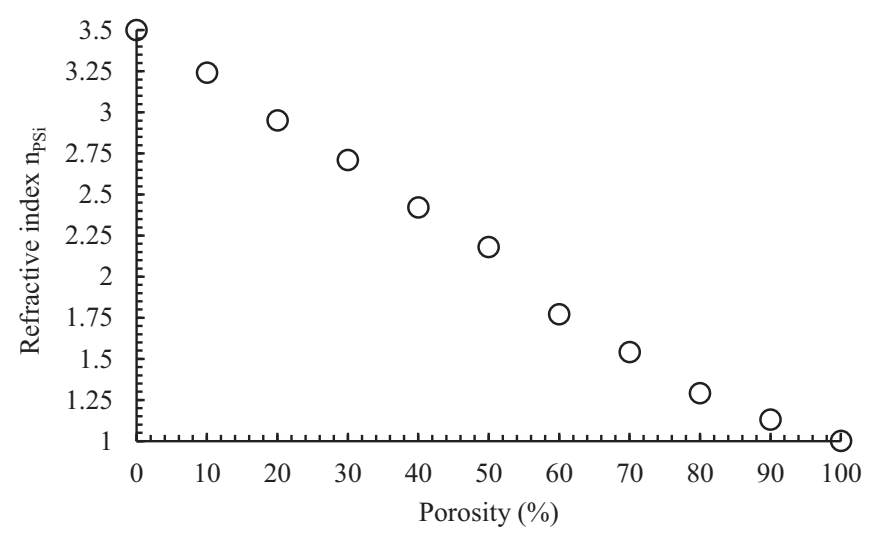

Figure 2. Calculated refractive index of PSi as a function of its porosity using the Bruggeman two media approximation.

It should be noted that Eq. 5 holds only for freshly produced PSi. However, due to the aging effect (oxidation), the impact of silicon dioxide $\left(n_{\mathrm{SiO}_{2}}\right)$ must also be considered (three media approach). ${ }^{2}$

Since an already formed PSi layer is unaffected by further electrochemical etching, ${ }^{42}$ fabrication of a multilayer PSi with a variety of refractive index profiles (one-dimensional photonic structures) is possible.

\section{Fabrication and Characterization of the Filter Wheel}

Design of the filter wheel.-Using a structured masking layer such as silicon nitride $\left(\mathrm{Si}_{3} \mathrm{~N}_{4}\right)$ and a very thin highly doped p-Si layer as the back electrode of a wafer, the current density provided through the back electrode into the p-Si wafer during the anodization can be locally adjusted by geometrical size and areal density of holes in the insulating $\mathrm{Si}_{3} \mathrm{~N}_{4}$ layer on the top face of the wafer. ${ }^{43}$ This approach can be employed to design a PSi based rugate filter wheel consisting of nine filter segments with different resonance peak wavelengths on a single wafer. For this purpose, 9 rectangular blocks (S1 - S9) with similar lengths $(13.5 \mathrm{~mm})$, but with different widths in a range of 1$3 \mathrm{~mm}$ with a step size of $0.25 \mathrm{~mm}$ were arranged in a circular format with a total diameter of $38 \mathrm{~mm}$ (see Fig. 3); the centerlines of the rectangular blocks through the origin point had angles of $\pm 42.2^{\circ}$ in respect to their adjacent blocks. In this way, each filter segment (e.g., S1) possesses a specific anodization or opening area (A1) and a specific effective surrounding masking area (M1 + M9) in which the effective

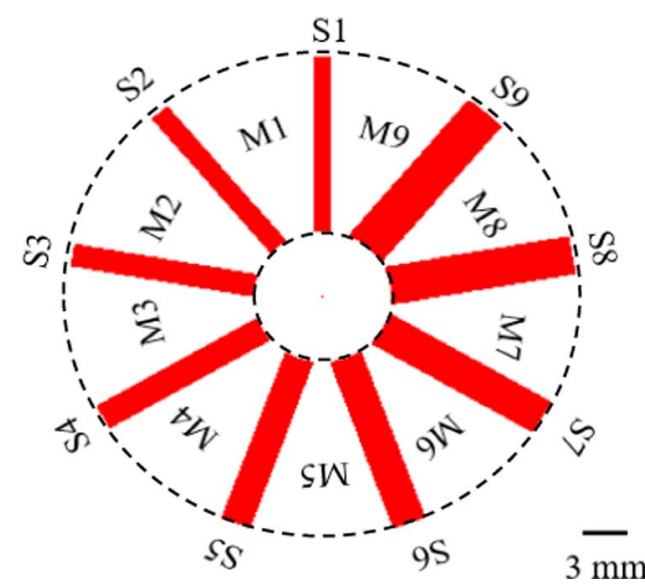

Figure 3. Designed filter wheel, consiting of nine filter segments arranged in a circular format with a diameter of $38 \mathrm{~mm}$. S stands for filter segment and $M$ stands for effective masking area. Dashed circles define the inner and outer boundaries of the filter segments and the effective masking areas. 
Anodization etch front at differente times :

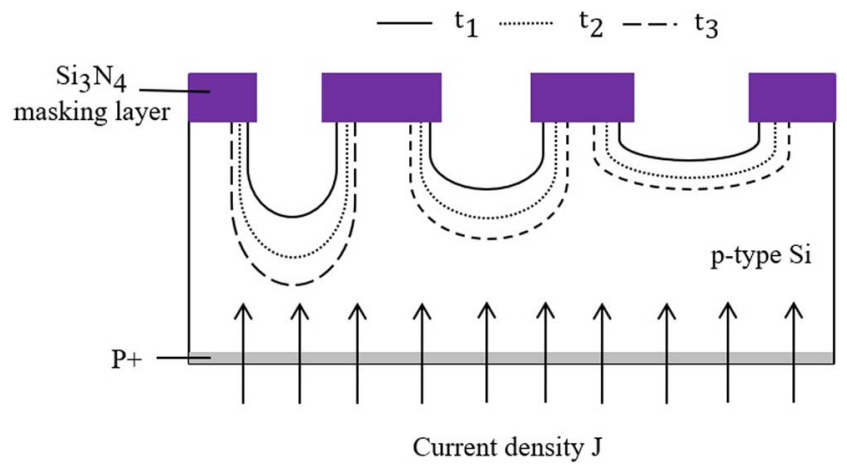

Figure 4. Cross-sectional view of etch fronts at different times for a structured $\mathrm{Si}$ wafer with different opening sizes (A1-A3) during anodization (the current squeezing effect).

masking areas follow the following relation: $\mathrm{M} 1>\mathrm{M} 2>\ldots>\mathrm{M} 8$ and M1 > M9 > M8. Thereby, the local current density which applies to each filter segment and consequently, formation rate and porosity of the PSi layers on each filter segment is locally altered depending on the area of the filter segment and its total surrounding masking area. The idea is based on the current squeezing effect (see Fig. 4). ${ }^{23,24}$ In other words, a higher current density flows through a smaller opening in a structured wafer with different opening sizes. The higher current density results in a larger formation rate and consequently, a thicker and higher porosity (lower refractive index) for a PSi layer. ${ }^{29,36}$ In this way, the resonance peak wavelength of a filter segment is varied since it is directly related to refractive index and thickness of its PSi layer (see Eq. 2).

Simulation of current density redistribution and current squeezing.-The local current density which affects a specific filter segment during the anodization for the designed filter wheel was investigated through an FEM model built in the COMSOL Multiphysics using the electric current interface of its AC/DC module. For this purpose, a double cell anodization tank (PSB4) from the Advance Micromachining Tools (AMMT) GmbH containing a 4-inch structured silicon nitride $\left(\mathrm{Si}_{3} \mathrm{~N}_{4}\right)$ wafer was considered as the wet etching system (see Fig. 5). The physical components, electrolyte, the structured silicon nitride wafer, and two platinum $(\mathrm{Pt})$ electrodes were created according to their real physical dimensions and specifications. The

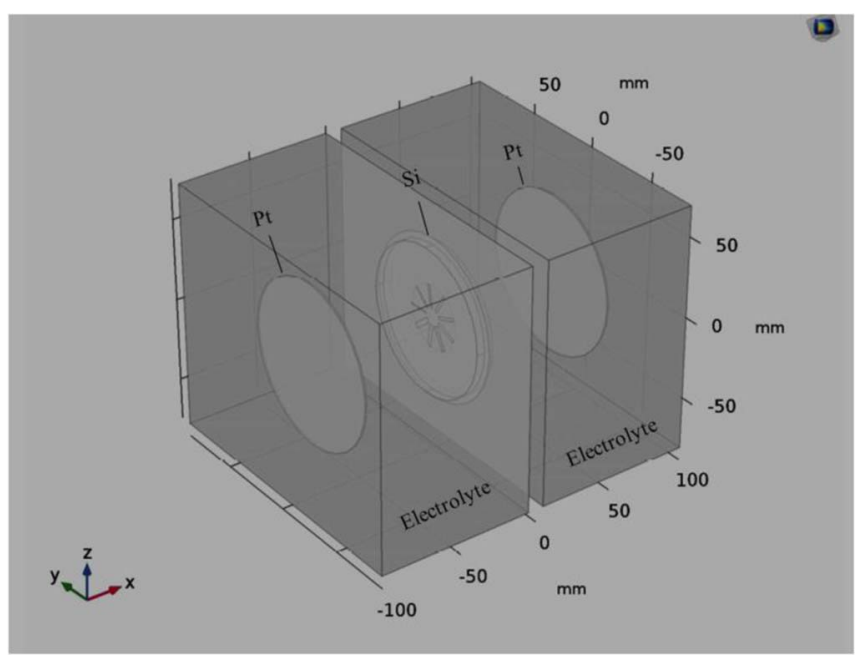

Figure 5. 3D schematic of the wet etching system modelled in COMSOL Multiphysics. a)

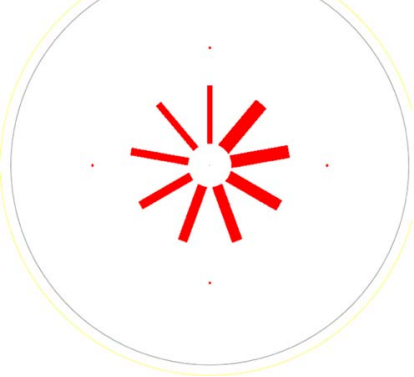

b)

Figure 6. Used positive masks to simulate and fabricate the designed rugate filterwheel on a wafer covered with silicon nitride layers: a) frontside mask, b) back side mask.

geometry of the electrolyte bath was drawn based on the internal dimension $\left(180 \times 150 \times 180 \mathrm{~mm}^{3}\right)$ of the PSB4 tank. The electrolyte was divided into two equal cells by the structured $\mathrm{Si}_{3} \mathrm{~N}_{4}$ wafer with a thickness of $500 \mu \mathrm{m}$. The electrolyte was defined as a nonsolid material with a relative permittivity of 11.7 and an electrical conductivity of $28.5 \mathrm{~S} / \mathrm{m}$, equivalent to the electrical property of $1: 1 \mathrm{v} / \mathrm{v} \% \mathrm{HF}$ :Ethanol solution at a temperature of $18^{\circ} \mathrm{C}$. Temperature of the bath was coupled to the electrical conductivity of the electrolyte since ionic conductivity of the electrolyte is related to its temperature. ${ }^{44}$ Two circular plates each with a diameter of $98 \mathrm{~mm}$ and a thickness of $1 \mathrm{~mm}$, which were symmetrically arranged to the symmetry axes of the $\mathrm{Si}$ wafer and were located $80 \mathrm{~mm}$ away from it on each cell were considered as the $\mathrm{Pt}$ electrodes. The electrodes were defined as solid materials with relative permittivities of 1 and electrical conductivities of $8.9 \times 10^{6} \mathrm{~S} / \mathrm{m}$, equivalent to the electrical properties of Pt. A circular plate with a diameter of $100 \mathrm{~mm}$ and a thickness of $500 \mu \mathrm{m}$ containing a centric circular opening with a diameter of $38 \mathrm{~mm}$ on its back side and the filter wheel segments opening on its front side (see Fig. 6) was used to represent the structured $\mathrm{Si}_{3} \mathrm{~N}_{4}$ wafer. The $\mathrm{Si}$ wafer was considered as a solid material with a relative permittivity of 11.7 and an electrical conductivity of $6.7 \times 10^{3} \mathrm{~S} / \mathrm{m}$, equivalent to electrical properties of highly doped $\mathrm{Si}$. The $\mathrm{Si}_{3} \mathrm{~N}_{4}$ masking layers were considered as void space without any physical definition in the model.

The Pt electrode, which faced the rear of the wafer (containing the circular opening) was grounded and the other one, which faced to the front of the wafer (containing the filter segments) was set as the terminal source providing a constant current of $10 \mathrm{~A}$ (equivalent to $8.81 \times 10^{5} \mathrm{~A} / \mathrm{m}^{2}$ for the designed filter wheel). The simulation results clearly showed redistribution and intensity of the current density flowing through each filter segment during the anodization step (see Fig. 7). The current squeezing which is responsible for the edge effects (pronounced etch rate near the mask edges) ${ }^{45,46}$ and its intensity with respect to size of the masking area, can be clearly seen in Figures 8 and 9. For instance, for the filter segment S9, the intensity of current density is higher at a side which has a higher masking area (M1) compared to the side with lower masking area (M9) due to current squeezing. This relation can be seen in other filter segments as well. The dependency of effective local current densities to the size of filter segments for the designed filter wheel during the anodization process is shown in Figure 9. The local current density entering a filter segment is highly dependent on the size of the filter segment. Increasing the width (since all segment have the same length) of filter segments results in a decrease in local current densities entering the filter segments.

It should be noted that the simulation neglects any influence of diffusion since a relatively thin PSi layer is assumed. However, diffusion effects must be considered in the case of thick PSi layers. ${ }^{25}$

Fabrication of the filter wheel.-A 4-inch boron-doped $<100>$ Czochralski (CZ) $10-20 \Omega / \square$ p-type Si wafer with a back side sheet resistance of $18.3 \pm 0.4 \Omega \mathrm{cm}$ (achieved by doping using a 


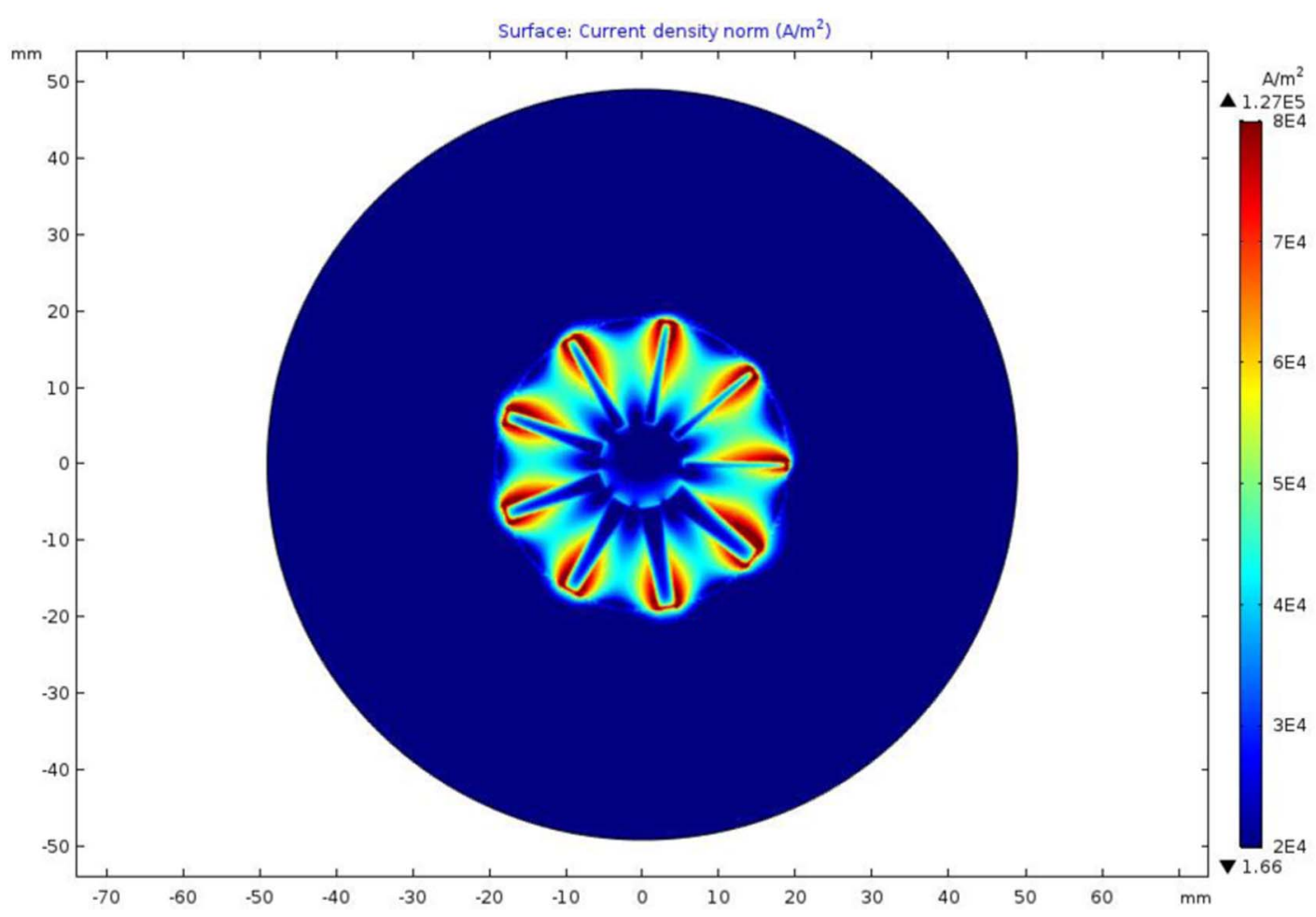

Figure 7. Redistribution and intensity of the current density during the anodization of a 4-inch structured Si wafer with the mask shown in Fig. 5, in a double cell anodization tank.

$\mathrm{BN}$ - thermal diffusion method) was patterned using $\mathrm{Si}_{3} \mathrm{~N}_{4}$ masking layers and direct laser writing lithography on both sides of the wafer to achieve defined and separated areas of filter segments. The thickness of the $\mathrm{Si}_{3} \mathrm{~N}_{4}$ masking layer was chosen to be around $180 \mathrm{~nm}$ in order to resist the hydrofluoric solution for at least 10 minutes ${ }^{47}$ and to suppress the building up of residual stress in the crystalline silicon. ${ }^{48}$ The structures shown in Fig. 6 were patterned on the front face and the rear face of the wafer to allow the passage of electrical current though the wafer during anodization. Anodic etching of the filter segments was then carried out in a double cell anodization tank (PSB4 by AMMT) by applying a sinusoidal current profile in 1.7 liter of $1: 1$ $\mathrm{v} / \mathrm{v} \% \mathrm{HF} /$ Ethanol solution with a constant temperature of $18.7^{\circ} \mathrm{C}$. A global peak resonance wavelength of $720 \mathrm{~nm}$ was considered to create

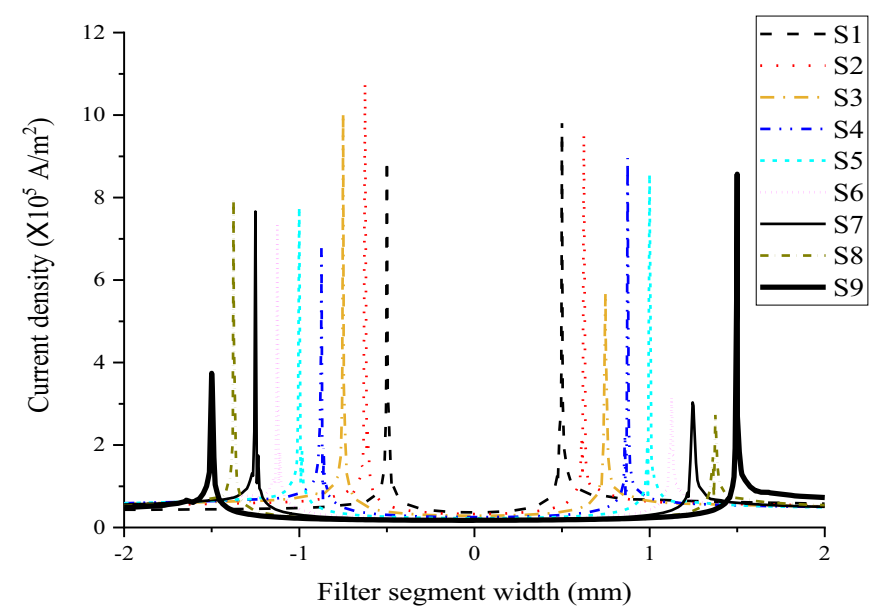

Figure 8. Simulated local current density impinging on each filter segment during the anodization. The origin defines the middle of the filter segments and the current density peaks occur at the edges of a specific filter segment. the sinusoidal current profile, and its required parameters $\left(n_{H}=1.41\right.$, $n_{L}=1.27, \Delta n=0.14, d_{H}=127.65 \mathrm{~nm}$, and $\left.d_{L}=141.73 \mathrm{~nm}\right)$ were obtained using Eq. 2. The sinusoidal current was oscillating between $20 \mathrm{~mA} / \mathrm{cm}^{2}$ and $70 \mathrm{~mA} / \mathrm{cm}^{2}$ with a frequency of $91.2 \mathrm{mHz}$ to gradually decrease the refractive index of the PSi layer from 1.41 to 1.27. The sinusoidal current profile was repeated 32 times (cycles) in order to generate a filter stack with 32 layers and to increase reflectance of the filters to about $99.5 \%$ (Eq. 3).

Filter wheel characterization results.-The actual view of the fabricated filter wheel exposed to ambient light and cross-sectional SEM images of filter segments 1 (the narrowest filter segment) and 9 (the widest filter segment) are shown in Figure 10. Since a crosssection was manually obtained by a diamond scriber, the cut did not go through the same plane for all 32 filter layers. Hence, the cross

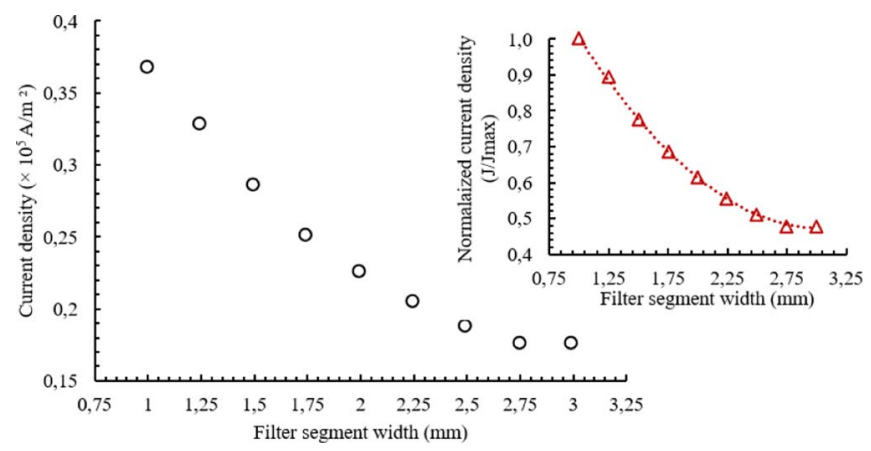

Figure 9. Simulated local current densities impinging on the center of each filter segment (position 0 in Fig. 8) as a function of the width of the filter segments. Inset: normalized current densities with respect to filter segment width. Normalized current densities were obtained by normalizing the current densities with respect to the maximum current density (the current density applied to the S1 segment). 

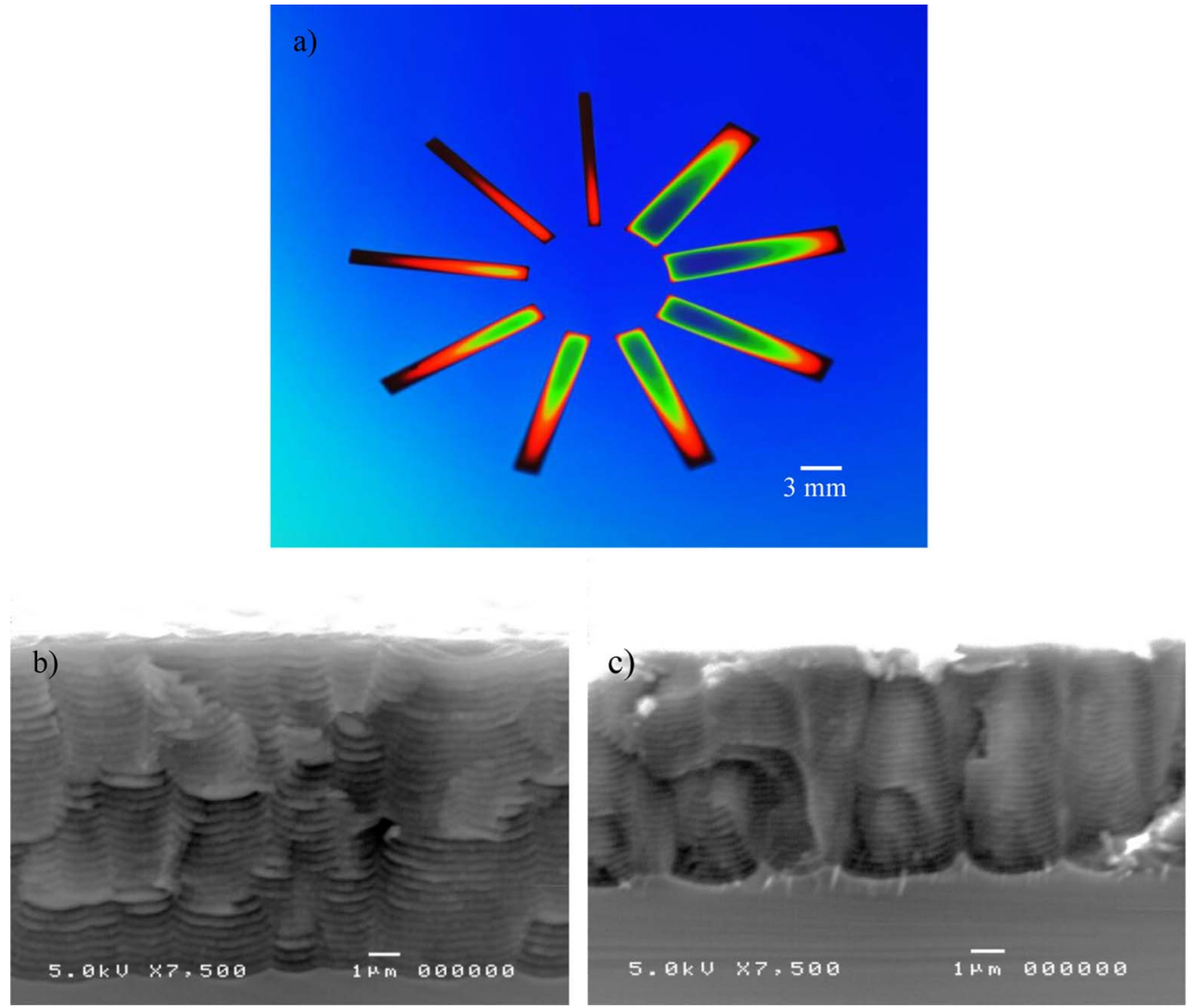

Figure 10. Fabricated rugate filter wheel: a) actual view of the filter wheel exposed to ambient light, b) cross-sectional SEM image of the filter segment 1 (the narrowest segment), and c) cross-sectional SEM image of the filter segment 9 (the widest segment).

section is not a continuous plane, and cut planes show slight steps between the filter layers. Filter segment colors at their central points are gradually altered from dark red to light green by increasing the width of the filter segments. The reflection spectrum of the filter wheel was measured by an AvaSpec ULS3648 spectrometer with a spot size of $1.5 \mathrm{~mm}$ and an integration time of $20 \mathrm{~ms}$. A spectrum was taken from the central point (half length) of each filter segment in order to compare the results and exclude the radial and edge effects of the anodization process on the filters' characteristics. For this purpose, a specially designed characterization setup consisting of a motorized rotary stage with a resolution of 0.05 degree and an adjustable $x-y-z$ axis fiber optic holder was used. The fabricated filter wheel provides a reflection spectrum with reflectance of $66-27 \%$ in a wavelength range of 570-820 nm (see Fig. 11). A filter segment's resonance peak wavelength shows a $2^{\text {nd }}$ degree polynomial relation to the width of the filter segment as is shown in Figure 12. Increasing the size of a filter segment resulted in a decrease in the reflecting wavelength of the filter segment. Additionally, it resulted in an increment in reflectance of the filter segment.

To determine the local anodization depths, the PSi layers were completely etched away in a $3 \% \mathrm{KOH}: \mathrm{H}_{2} \mathrm{O}$ solution at room temperature for a duration of 1 minute. The etch depth, which represents the PSi thickness in a specific segment, was measured for each filter segment using a Dektak 150 surface profiler from Veeco Instruments Inc. Measurements were done by line scans over the width of a filter segment (measuring position: half length). Topographical scans of all etched cavities showed a convex shape with sharp minima where the etch depth increased from the center of a structure to its borders (see Fig. 13 inset). However, the etch depths were deeper at those sides which had higher masking areas (see Fig. 3). The maximum etch depths were considered and plotted as a function of the width of the filter segments (see Fig. 13). Results show that the PSi thickness is moderately decreasing with increasing width of a filter segment.
The narrowest filter segment ( $\mathrm{S} 1,1 \mathrm{~mm}$ wide) resulted in the thickest PSi (13.8 $\mu \mathrm{m}$ thick), and the widest filter segment ( $\mathrm{S} 9,3 \mathrm{~mm}$ wide) resulted in the thinnest PSi ( $9.7 \mu \mathrm{m}$ thick); these characteristics can be also seen in Figures 10b and 10c. Hence, this design allows the relative variation of the anodization rate of about $25 \%$.

Multispectral imaging system.-The PSi rugate filter wheel presented above can be used to enable a multispectral imaging system based on the wavelength scanning method. Using this method, the 3D data cube containing the spatial and spectral information can be constructed by stacking 2D images of the target, where each image is acquired at a different wavelength. Figure 14 shows a schematic representation of how this can be realized using the PSi filter wheel.

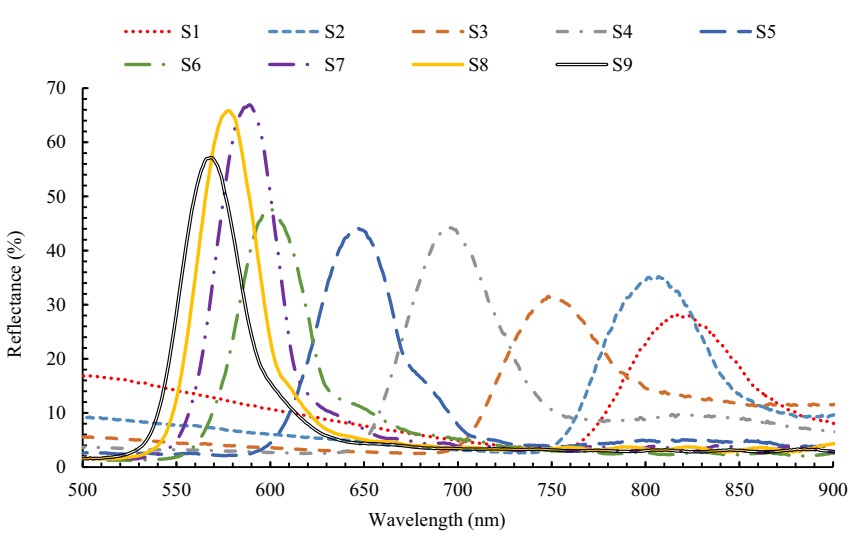

Figure 11. Reflectance of the filter wheel segments as a function of wavelength. 


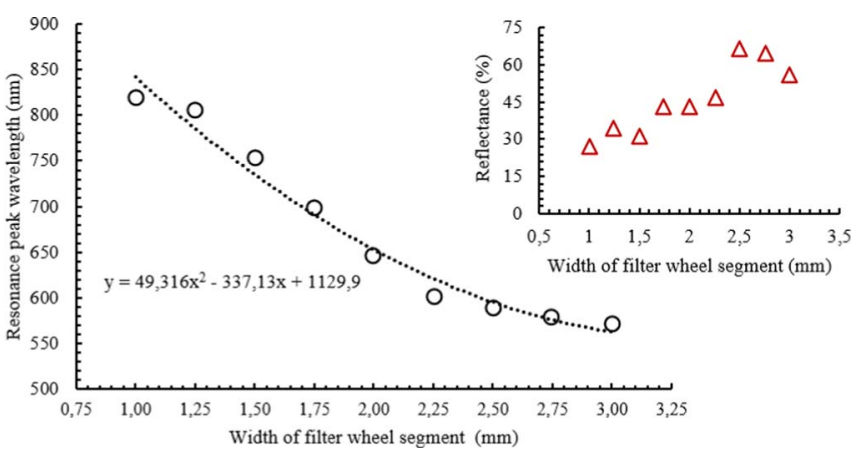

Figure 12. Resonance peak wavelength as a function of filter segment width, measured at the center of each segment. Inset: reflectivity of a filter segment as a function of filter segment width.

The incoming light from the target is focused on a filter segment of the wheel. The image reflected from the filter carries the spectral information for the wavelength associated with the filter segment. The image is projected on the imaging sensor by collimating and imaging lenses, and then recorded as one plane of the data cube. By rotating the filter wheel to scan across the filter segments, an image of the target is recorded at each wavelength and the data cube is constructed. Miniaturization and integration of the developed filter wheel into a portable MOEMS system will be later discussed in a separate paper.

\section{Discussion}

The simulation results and experimental results of the filter wheel agree well with the theory and confirm the feasibility of the proposed approach and thus the possibility of breaking the symmetry of the anodization process. Simulation results show that a local current density which applies on a filter segment during the anodization is proportionally related to the opening size (opening area) of the filter segment. In the proposed filter wheel where all filter segments have the same length, areas of filter segments are altered by their widths. In this case, local current densities impinging on filter segments decrease proportionally with increasing width of the filter segments. Since porosity and formation rate of a PSi layer is defined by the amplitude of applied current density, a decrement in local current density results in a decrease in porosity and formation rate of PSi. ${ }^{49-51}$ This results in a slight increment in refractive index, and a slight decrement in thickness of the PSi layer and consequently, a shift in reflecting resonance peak wavelengths of the filter segments.

Although a global peak resonance wavelength of $720 \mathrm{~nm}$ was considered to create the initial sinusoidal current profile (in which the current was sinusoidally oscillated between $20 \mathrm{~mA} / \mathrm{cm}^{2}$ and $70 \mathrm{~mA} / \mathrm{cm}^{2}$ with a frequency of $91.2 \mathrm{mHz}$ during anodization), the fabricated filter wheel provided a reflection spectrum in a wavelength range of 570

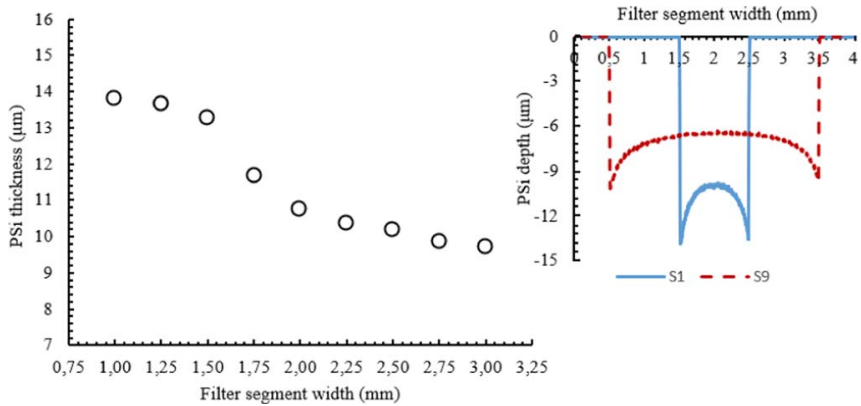

Figure 13. PSi thickness as a function of filter segment width measured by line scans over the width of the filter segments (measuring position: half length). Inset: line scan results over the width of filter segments S1 and S9 obtained by a Dektak surface profiler.

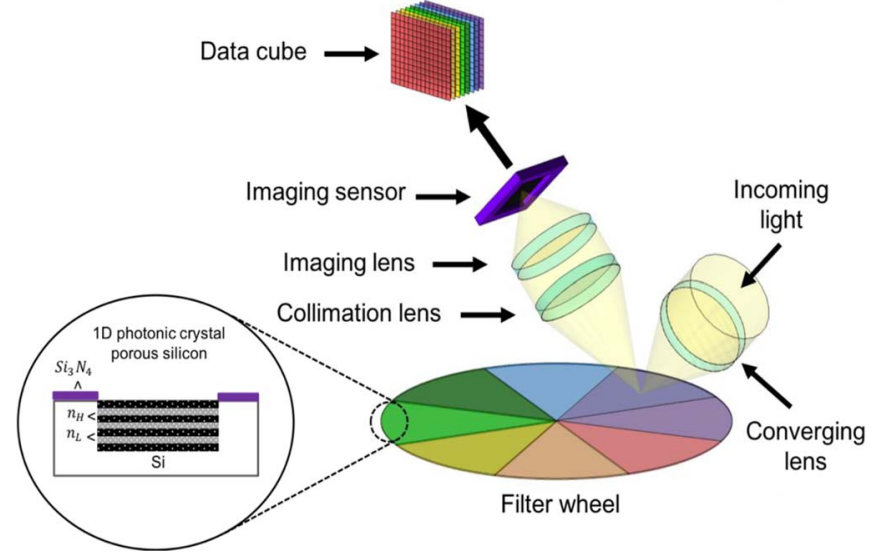

Figure 14. Concept of multispectral imaging system based on wavelength scanning using a rotating filter wheel.

$820 \mathrm{~nm}$ with different reflectance peak values in the range of $66-27 \%$. This confirms that the local current density impinging on an individual filter segment did not follow the initial set current density profile due to the filter wheel pattern. The peak-to-peak amplitude of local oscillating current densities exceeded the initial set current density in the filter segments S1 - S3 (providing wavelengths of $820-750 \mathrm{~nm}$ ) and was diminished in the filter segments S4 - S9 (providing wavelengths of $700-570 \mathrm{~nm})$, while their frequencies $(91.2 \mathrm{mHz})$ had been maintained constant. The effect can be explained well when considering a uniform charge flow over the sample area coming from the rear of the wafer. The localization of an applied current density with a front face insulating layer makes the charge flow to squeeze through the limited opening regions. The smaller the opening regions, the more concentration of the charge is achieved, resulting in a higher current density, and hence etch rate. The dependence of local current density to size (area) of an opening (filter segment) indicates that there is indeed redistribution of current density between the openings. This is either due to an increase in the electrical resistance of the diffuse ionic layer in the electrolyte or due to a decrease in the electrical resistance in the substrate for deeper structures. ${ }^{25}$ Indeed, the locally modified current density had altered $d_{H}, d_{L}$, and the ratio between $n_{H}$ and $n_{L}$ of the PSi layer in each filter segment. This disrupted the $n_{H} d_{H}=n_{L} d_{L}$ condition in every filter segment and resulted in a relative shift in the peak resonance wavelength and a non-linear change in the resolution and reflectance of the filter segments. The non-linearity in the resolution of the filter wheel in respect to the filter segment width can be due to resistivity range of the wafer $(10-20 \Omega \mathrm{cm})$ since the radial and axial resistivity variations on the wafer can create different etch rates at different regions of the wafer. Using highly doped silicon wafers (e.g., 0.01-0.02 $\Omega \mathrm{cm}$ ) can improve the nonlinearity in resolution since the radial and axial resistivity variations in such wafers are very small. However, the non-linearity in reflectance can be suppressed by increasing number of filter stack according to Eq. 3 .

The preliminary results show that the proposed approach can be employed to adjust a filter wheel with a defined peak resonance wavelength range and resolution by adjusting the size and number of the filter segments and the initial current density profile. The approach can be used to provide low cost filter wheels suitable for wavelength scanning multispectral imaging systems. Miniaturization of the filter wheel is also possible since the down scaling can be done by current density adjustment for a specific wafer resistivity range and type of electrolyte used.

\section{Conclusions}

A porous silicon based rugate filter wheel containing nine filter segments in the visible wavelength range $(520-820 \mathrm{~nm})$ suitable for multispectral imaging systems was presented. The filter wheel 
segments were structured on a low doped p-type silicon wafer covered with insulating silicon nitride layers and fabricated using the anodization technique. Specific characteristics of each filter segment was altered by varying of a local current density flowing through each filter segment through the patterned and surrounded masking areas (the current squeezing effect). A finite element model made in COMSOL Multiphysics was also presented to study the redistribution of current density and the current squeezing during the anodization.

\section{Acknowledgments}

The authors thank the Ministry of Science, Research, and the Art at the state of Baden-Württemberg, Germany for providing the financial support in the framework program of "Upper-Rhine Research Alliance for the Technical Principles of Sustainability".

\section{ORCID}

Shervin Keshavarzi (D) https://orcid.org/0000-0002-2003-6700

\section{References}

1. F. A. Harraz, Sensors and Actuators B: Chemical, 202, 897 (2014).

2. M. S. Salem, M. J. Sailor, F. A. Harraz, T. Sakka, and Y. H. Ogata, Journal of Applied Physics, 100(8), 83520 (2006)

3. J. Volk, T. Le Grand, I. Bársony, J. Gombköto, and J. J. Ramsden, J. Phys. D: Appl. Phys., 38(8), 1313 (2005).

4. R. C. Erson, R. S. Muller, and C. W. Tobias, Sensors and Actuators A: Physical, 23(1-3), 835 (1990).

5. S. Zangooie, R. Jansson, and H. Arwin, Journal of Applied Physics, 86(2), 850 (1999)

6. J. M. Buriak, Philosophical transactions. Series A, Mathematical, physical, and engineering sciences, 364(1838), 217 (2006).

7. S. E. Létant, B. R. Hart, S. R. Kane, M. Z. Hadi, S. J. Shields, and J. G. Reynolds, Adv. Mater, 16(8), 689 (2004).

8. L. de Stefano, P. Arcari, A. Lamberti, C. Sanges, L. Rotiroti, I. Rea, and I. Rendina Sensors (Basel, Switzerland), 7(2), 214 (2007).

9. B. Guan, A. Magenau, S. Ciampi, K. Gaus, P. J. Reece, and J. J. Gooding, Bioconjugate chemistry, 25(7), 1282 (2014).

10. A. Janshoff, K.-P. S. Dancil, C. Steinem, D. P. Greiner, V. S.-Y. Lin, C. Gurtner K. Motesharei, M. J. Sailor, and M. R. Ghadiri, J. Am. Chem. Soc., 120(46), 12108 (1998).

11. V. Torres-Costa, F. Agulló-Rueda, R. J. Martín-Palma, and J. M. Martínez-Duart, Optical Materials, 27(5), 1084 (2005).

12. S. Li, D. Hu, J. Huang, and L. Cai, Nanoscale Research Letters, 7(1), 79 (2012).

13. H. Ouyang, M. Christophersen, R. Viard, B. L. Miller, and P. M. Fauchet, Adv. Funct. Mater., 15(11), 1851 (2005).

14. G. Rong, A. Najmaie, J. E. Sipe, and S. M. Weiss, Biosensors \& bioelectronics, 23(10), 1572 (2008).

15. C. Jamois, C. Li, E. Gerelli, Y. Chevolot, V. Monnier, R. Skryshevskyi, R. Orobtchouk, E. Souteyrand, and T. Benyattou, in SPIE Photonics Europe, H. R. Míguez, S. G. Romanov, L. C. Andreani, and C. Seassal, Editors, p. 77130U, SPIE (2010).

16. J. D. Ryckman, M. Liscidini, J. E. Sipe, and S. M. Weiss, Appl. Phys. Lett., 96(17), $171103(2010)$
17. S. Ilyas, T. Böcking, K. Kilian, P. J. Reece, J. Gooding, K. Gaus, and M. Gal, Optical Materials, 29(6), 619 (2007).

18. A. Garcia Sega, B. H. King, J. Y. Lee, M. J. Sailor, and G. M. Miskelly, ACS nano, 7(9), 7785 (2013)

19. A. Nahidi, W. E. Roper, and R. B. Gomez, in AeroSense 2003, N. L. Faust and W. E. Roper, Editors, p. 167, SPIE (2003).

20. G. Lu and B. Fei, Journal of biomedical optics, 19(1), 10901 (2014).

21. H. Huang, L. Liu, and M. O. Ngadi, Sensors (Basel, Switzerland), 14(4), 7248 (2014).

22. F. Koenig and W. Praefcke, in SYBEN-Broadband European Networks and Electronic Image Capture and Publishing, J. Bares, Editor, p. 34, SPIE (1998).

23. G. Korotcenkov, Editor, Porous silicon: From formation to application, Boca Raton, Fla., CRC Press (2016).

24. G. Lammel, S. Schweizer, and P. Renaud, Optical microscanners and microspectrometers using thermal bimorph actuators, Boston, Dordrecht, London, Kluwer Academic Publishers (2002).

25. A. Ivanov, Silicon Anodization as a Structuring Technique: Literature Review, Modeling and Experiments, Wiesbaden, Springer Fachmedien Wiesbaden (2017).

26. L. Tsakalakos, Nanotechnology for photovoltaics, Boca Raton, Taylor \& Francis (2010).

27. N. Perelman and I. Averbukh, Journal of Applied Physics, 79(6), 2839 (1996).

28. E. Lorenzo, C. J. Oton, N. E. Capuj, M. Ghulinyan, D. Navarro-Urrios, Z. Gaburro, and L. Pavesi, Applied optics, 44(26), 5415 (2005).

29. G. Korotcenkov, Editor, Optoelectronics, microelectronics, and energy technology applications, Boca Raton, FL, London, New York, CRC Press Taylor \& Francis Group (2016)

30. B. G. Bovard, Applied optics, 32(28), 5427 (1993).

31. N. Ishikura, M. Fujii, K. Nishida, S. Hayashi, and J. Diener, Infrared Physics \& Technology, 53(4), 292 (2010).

32. N. Ishikura, M. Fujii, K. Nishida, S. Hayashi, and J. Diener, Optics express, 16(20), 15531 (2008)

33. M. S. Salem, M. J. Sailor, K. Fukami, T. Sakka, and Y. H. Ogata, Journal of Applied Physics, 103(8), 83516 (2008).

34. R. A. Rakhimov, E. V. Osipov, D. S. Dovzhenko, I. L. Martynov, and A. A. Chistyakov, J. Phys.: Conf. Ser., 737, 12026 (2016).

35. A. Kovacs, P. Jonnalagadda, X. Y. Meng, and U. Mescheder, in 2009 IEEE Sensors, p. 21.

36. S. Keshavarzi, F. Lima, W. Kronast, and U. Mescheder, J. Microelectromech. Syst., 27(2), 250 (2018).

37. G. X. Zhang, in Modern Aspects of Electrochemistry, C. G. Vayenas, R. E. White, and M. E. Gamboa-Adelco, Editors, p. 65, Boston, MA, Springer US (2006).

38. H. Sohn, in Handbook of Porous Silicon, L. Canham, Editor, p. 1, Cham, Springer International Publishing (2021)

39. D. A. G. Bruggeman, Ann. Phys., 416(7), 636 (1935).

40. O. Zhuromskyy, Crystals, 7(1), 1 (2017).

41. M. S. Salem, A. M. Abdelaleem, A. A. El-Gamal, and M. Amin, Journal of Applied Physics, 121(1), 13108 (2017).

42. I. Ronga, J. Electrochem. Soc, 138(5), 1403 (1991)

43. U. M. Mescheder, A. Kovacs, A. Fahad, and R. Huster, in 2002 2nd IEEE Conference on Nanotechnology, p. 33.

44. E. G. Hill and A. P. Sirkar, Proceedings of the Royal Society A: Mathematical, Physical and Engineering Sciences, 83(560), 130 (1909).

45. A. Ivanov, A. Kovacs, and U. Mescheder, Phys. Status Solidi A, 208(6), 1383 (2011)

46. H.-S. Kim, K. Chong, and Y.-H. Xie, Appl. Phys. Lett., 83(13), 2710 (2003).

47. G. Lammel and P. Renaud, Sensors and Actuators A-Physical, 85(1-3), 356 (2000).

48. A. Splinter, O. Bartels, and W. Benecke, Sensors and Actuators B: Chemical, 76(1-3), 354 (2001).

49. Y. Arita, J. Electrochem. Soc., 124(2), 285 (1977).

50. R. Herino, J. Electrochem. Soc., 134(8), 1994 (1987).

51. Y. Watanabe, J. Electrochem. Soc., 122(10), 1351 (1975). 